\title{
Perceived Stigma and Help-Seeking Behavior: Longitudinal Evidence From the Healthy Minds Study
}

\author{
Ezra Golberstein, Ph.D., \\ Department of Health Care Policy, Harvard Medical School, 180 Longwood Ave., Boston, MA 02115 \\ (golberstein@hcp.med.harvard.edu). \\ Daniel Eisenberg, Ph.D., and Sarah E. Gollust, Ph.D. \\ Robert Wood Johnson Foundation Health and Society Scholar at the University of Pennsylvania, \\ Philadelphia.
}

\begin{abstract}
Objective-Despite considerable policy interest in the association between perceived public stigmatization of mental illness and use of mental health services, limited empirical evidence, particularly from longitudinal data, documents this relationship. This study used longitudinal data to estimate the association between perceived public stigmatization and subsequent mental health care seeking. Methods: A Web-based survey was used to collect data from a random sample of undergraduate and graduate students at a university at baseline and two years later $(\mathrm{N}=732)$. Logistic regression models assessed the association between students' perceived public stigma at baseline and measures of subsequent help seeking for mental health problems (perceived need for help and use of mental health services) at follow-up. Results: No significant associations were found between perceived public stigma and help-seeking behavior over the two-year period. Conclusions: In this population of college students, perceived stigma did not appear to pose a substantial barrier to mental health care.
\end{abstract}

Reducing stigma is one of the major policy approaches proposed to reduce levels of unmet need for mental health services (1). This policy focus is understandable for two reasons. First, the stigma associated with mental illness by the public has remained persistent, with no apparent improvements over the past ten years (2). Second, it seems logical that individuals who believe that the public stigmatizes mental illness or mental health services might forgo treatment in order to avoid being labeled as a member of a stigmatized group (3).

Despite these compelling reasons to consider stigma an important barrier to mental health care, there is limited empirical evidence that stigma actually affects use of mental health services

(4). In this report we focus on perceived public stigma, one of multiple dimensions of stigma (3). Perceived stigma was associated with lower medication adherence in a longitudinal study of a clinical sample, but less is known about the association between perceived stigma and use of mental health services in nonclinical samples (5). Prior research addressed this topic using cross-sectional data from a representative sample of students at a large public university (4). Among students under age 22, higher perceived stigma was associated with a lower probability

\footnotetext{
(C) 2009 American Psychiatric Association

The official published article is available online at http://ps.psychiatryonline.org/cgi/content/full/60/9/1254?

maxtoshow $=\&$ hits $=10 \&$ RESULTFORMAT $=1 \&$ author $1=$ golberstein $\&$ andorexacttitle=and $\&$ andorexacttitleabs=and $\&$ andorexactfullte $\mathrm{xt}=$ and \&searchid=1\&FIRSTINDEX=0\&sortspec=relevance\&resourcetype=HWCIT .

The authors report no competing interests.
} 
of perceiving a need for help, but no significant association was found between perceived stigma and perceived need for help among older students, nor were there any significant associations for any student group between perceived stigma and actual service use.

In that study's cross-sectional analysis, however, the relationship between perceived stigma and service use was likely to reflect not only the effect of perceived stigma on service use but also the reverse, the effect of service use on perceived stigma. In this study, we improved upon prior research by using longitudinal data to test for an association between perceptions of stigma and subsequent help-seeking behavior.

\section{Methods}

Our data are from the Healthy Minds Study, a Web-based survey of a random sample of undergraduate and graduate students at a large public university (4). In the first wave of the survey, 2,782 students who were invited by e-mail to participate completed the survey in October 2005 (55.4\% response rate). Nonresponse weighting was used to ensure that the sample was representative of the total student population at the university.

Two years later, in October 2007, we invited the 1,272 baseline respondents who were still enrolled at the university to participate in a follow-up survey. A total of $747(59 \%)$ completed the survey. We constructed nonresponse weights using data on mental health and other variables collected at baseline so that the follow-up sample would be representative of all 1,272 students who were eligible for the analysis. The analyses included all students who responded to both the baseline and follow-up surveys and who had complete data for the measures used in the analyses $(\mathrm{N}=732)$. All participants gave informed consent, and the study was approved by the university's Health Sciences Institutional Review Board.

The dependent variables were binary measures of help-seeking behavior that were assessed in the follow-up survey. The first dependent variable was whether a respondent reported perceiving a need for help with mental health problems in the past two years (since the baseline interview). The second dependent variable was whether a respondent reported using any mental health services - that is, psychotropic medications and psychotherapy-in the past two years.

The main independent variable was the respondent's perceptions of the public's stigmatizing attitudes toward mental health service use reported in the 2005 survey. We adapted this measure from the five-item Stigma Scale for Receiving Psychological Help, which is one of the only brief measures of perceived stigma that has been validated in prior research $(6,7)$. The scale has been described in more detail elsewhere (4). Possible scores range from 0 , lowest perceived stigma, to 15 , highest perceived stigma.

We estimated two logistic regression models to test for an association between perceived stigma in 2005 and help seeking over the subsequent two years. The first model used perceived need for help as the dependent variable. The second model used mental health service use as the dependent variable and included perceived need for help as a covariate. All models also included the following baseline covariates: dummy variables for gender, age, race-ethnicity, U.S. or international student, family's financial situation while the student was growing up, and depression severity, which was measured by the Patient Health Questionnaire (PHQ) (8). All analyses were conducted with Stata, version 10.1, and were weighted to adjust for nonresponse.

\section{Results}

Sociodemographic and clinical characteristics and levels of perceived stigma among the 732 students with complete data in the 2007 follow-up were similar to those in the baseline sample 
in 2005 (complete data are available from the authors upon request). Briefly, there were 308 men $(47.3 \%)$ in the 2007 sample. 457 students $(60.5 \%)$ were white, $22(3.8 \%)$ were black, 25 (3.4\%) were Hispanic or Latino, 156 (22.1\%) were Asian or Pacific Islander, and 72 (10.5\%) were of other racial-ethnic background. 314 (46.5\%) of the students were age 18-22, 120 (16.2\%) were age 23-25, 196 (24.2) were age 26-30, and $102(13.1 \%)$ students were 31 or older.

As shown in Table 1, no significant association was found between perceived stigma in 2005 and perceived need for help or mental health service use over the next two years. We obtained the same results when we restricted the sample to respondents with a probable mental health problem, as defined by a positive screen on the PHQ depression or anxiety scales in either 2005 or 2007 and when we stratified the sample by age (18-22 age group versus those older than 22) (results available upon request).

\section{Discussion}

To enhance empirical evidence of the influence of perceived stigma on help-seeking behavior, we collected longitudinal data to determine whether a student's baseline perception of public stigmatization of mental health service use was correlated with the student's perception of a need for help and with service use over the next two years. We found no evidence that perceived stigma was associated with subsequent mental health help-seeking behavior in the student population. This finding bolsters a similar finding from a previous cross-sectional study, because we can now rule out the possibility that the null result is due to reciprocal causality.

The study reported here has notable limitations. First, our results do not necessarily represent the causal effect of perceived stigma on help seeking, because unobserved variables, such as knowledge or motivation related to treatment, may confound the longitudinal relationship between perceived stigma and subsequent help seeking. Second, our measure of perceived public stigmatization of mental health service use did not capture other aspects of stigma, such as one's own stigmatizing attitudes toward mental illness. Two recent survey studies of college students, although limited by their cross-sectional nature, suggest that personal or self-stigma may be a more important barrier to help seeking than perceived public stigma $(9,10)$. Third, our study was restricted to students at a single university, and the results may not generalize to other groups.

\section{Conclusions}

To the extent that our estimates approximate true causal relationships, they suggest that reducing perceptions of public stigma may not lead to significant increases in help-seeking behavior, at least among college students. This does not imply that reducing stigma is unimportant, because other facets of stigma may affect help seeking and stigma may have other negative consequences, such as reduced self-esteem or impaired social relationships. Additional longitudinal research is needed to resolve whether these findings apply to other populations and whether other factors, such as stigmatizing attitudes or self-stigma, represent more significant barriers to services.

\section{Acknowledgments}

The authors gratefully acknowledge financial support from training grant T32-MH019733 from the National Institute of Mental Health and additional support from the Blue Cross Blue Shield Foundation of Michigan (grant \#1069) and the Robert Wood Johnson Foundation Health and Society Scholars Program at the University of Pennsylvania. The authors also acknowledge the following sponsors at the University of Michigan: the Comprehensive Depression Center (Innovation Fund), a McNerney Award, School of Public Health, the Office of the Vice-President for Research, and Rackham Graduate School. They thank Scott Crawford, M.A., and the Survey Sciences Group for fielding and coordinating the survey. 


\section{References}

1. Mental Health: A Report of the Surgeon General. US Department of Health and Human Services, US Public Health Service; Rockville, Md: 1999.

2. Schnittker J. An uncertain revolution: why the rise of a genetic model of mental illness has not increased tolerance. Social Science and Medicine 2008;67:1370-1381. [PubMed: 18703264]

3. Corrigan P. How stigma interferes with mental health care. American Psychologist 2004;59:614-625. [PubMed: 15491256]

4. Golberstein E, Eisenberg D, Gollust SE. Perceived stigma and mental health care seeking. Psychiatric Services 2008;59:392-399. [PubMed: 18378838]

5. Sirey JA, Bruce ML, Alexopolous GA, et al. Perceived stigma and patient-rated severity of illness as predictors of antidepressant drug adherence. Psychiatric Services 2001;52:1615-1620. [PubMed: 11726752]

6. Pyne JM, Kuc EJ, Schroeder PJ, et al. Relationship between perceived stigma and depression severity. Journal of Nervous and Mental Disease 2004;192:278-283. [PubMed: 15060401]

7. Komiya N, Good GE, Sherrod NB. Emotional openness as a predictor of college students' attitudes toward seeking psychological help. Journal of Counseling Psychology 2000;47:138-143.

8. Spitzer RL, Kroenke K, Williams JBW, et al. Validation and utility of a self-report version of PRIMEMD: the PHQ primary care study_Primary Care Evaluation of Mental Disorders, Patient Health Questionnaire. JAMA 1999;282:1737-1744. [PubMed: 10568646]

9. Vogel DL, Wade NG, Hackler AH. Perceived public stigma and the willingness to seek counseling: the mediating roles of self-stigma and attitudes toward counseling. Journal of Counseling Psychology 2007;54:40-50.

10. Eisenberg D, Downs M, Golberstein E, et al. Stigma and help-seeking for mental health among college students. Medical Care Research and Review. in press. 


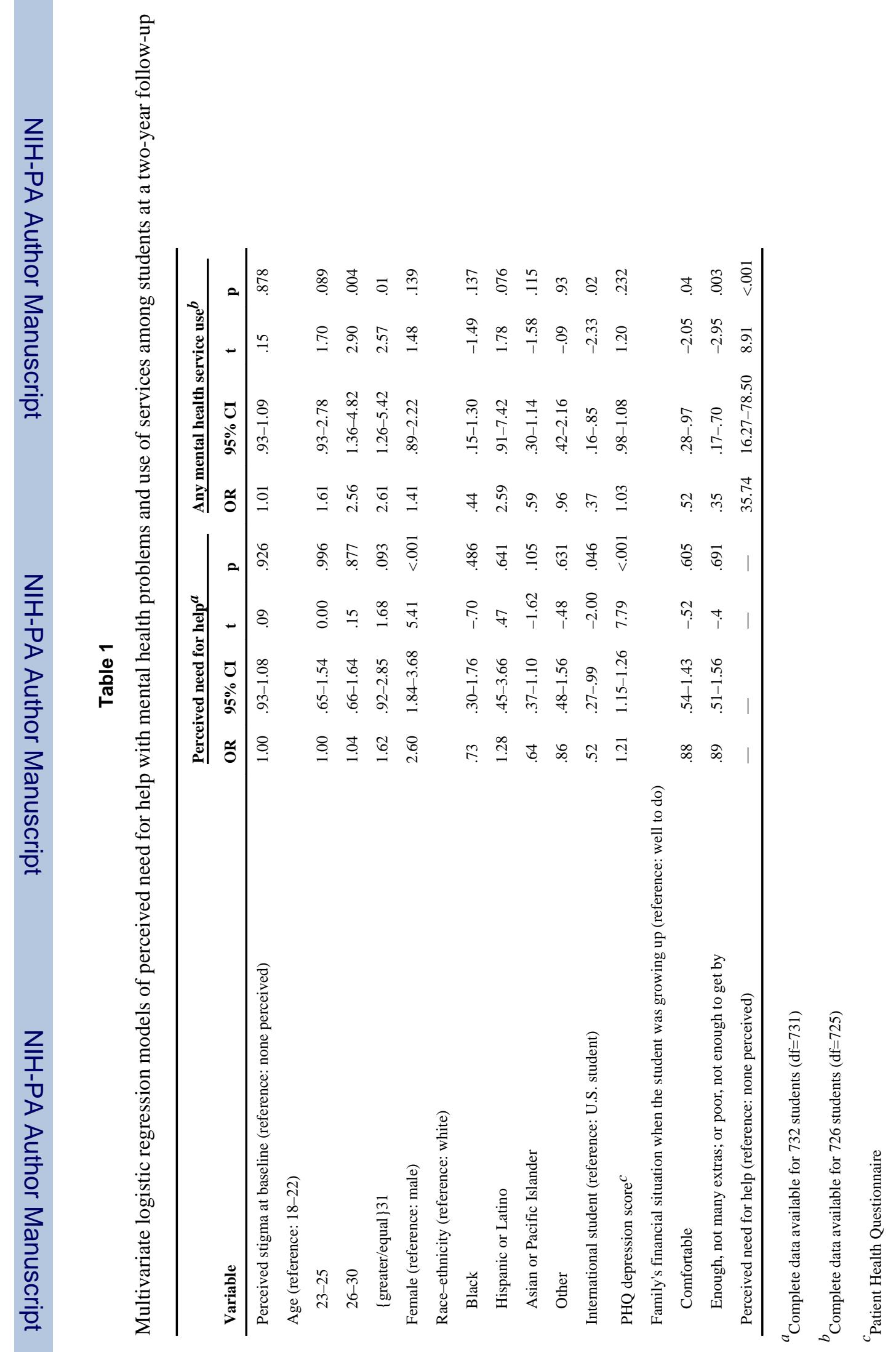

Psychiatr Serv. Author manuscript; available in PMC 2010 November 9. 